\title{
Hipoalbuminemia como predictor de mortalidad en sepsis de origen abdominal
}

\author{
Hypoalbuminemia as a predictor of mortality in abdominal sepsis
}

\author{
Eric M. Saucedo-Moreno*, Enrique Fernández-Rivera y José A. Ricárdez-García
}

Servicio de Cirugía General, Hospital Ángeles MOCEL, Ciudad de México, México

\section{Resumen}

Antecedentes: Un valor de albúmina < $35 \mathrm{~g} / \mathrm{l}$ (hipoalbuminemia) ha demostrado ser un parámetro para predecir mortalidad y morbilidad. Método: Estudio observacional, prospectivo, en un periodo de 12 meses, en pacientes con diagnóstico de sepsis de origen abdominal a quienes se dividió en dos grupos según las cifras de albúmina (corte: $3.5 \mathrm{~g} / \mathrm{dl}$ ) para valorar la mortalidad en ambos grupos Resultados: Estudiamos 23 pacientes ingresados a la unidad de terapia intensiva. La media de albúmina fue de $2.77 \mathrm{~g} / \mathrm{dl}$ ( \pm 0.71). Al calcular la odds ratio $(O R)$ identificamos un riesgo 23 veces mayor de fallecer al presentar hipoalbuminemia en comparación con el grupo con albúmina normal $(O R=23.3$; intervalo de confianza del $95 \%$ [IC 95\%]: 1.948 a 279.42). La media de los valores de albúmina para los pacientes que fallecieron fue de $2.04 \mathrm{~g} / \mathrm{dl}$ ( \pm 0.31 ) vs. a $3.03 \mathrm{~g} / \mathrm{dl}$ ( \pm 0.35) para el otro grupo (IC 95\%: -1.551 a $-0.416 ; p=0.02)$ ]. Aunque no valoramos la morbilidad, identificamos cierta tendencia a un mayor tiempo de estancia en la unidad de terapia intensiva, lo que se acompaña de mayor riesgo de complicaciones y de un mayor riesgo de muerte. Conclusión: La hipoalbuminemia representa un predictor de mortalidad en los pacientes con sepsis abdominal.

Palabras clave: Hipoalbuminemia. Sepsis. Sepsis abdominal.

\begin{abstract}
Background: A level < $35 \mathrm{~g} / \mathrm{L}$ of albumin (hypoalbuminemia) has been determined as a parameter to predict mortality and morbidity. Method: Prospective observational study, in a period of 12 months, to patients diagnosed with sepsis of abdominal origin, they are divided into two groups based on albumin levels (cut: $3.5 \mathrm{~g} / \mathrm{dL}$ ) to assess mortality between both groups. Results: We studied 23 patients admitted to the intensive care unit. The mean albumin was $2.77 \mathrm{~g} / \mathrm{dL}( \pm 0.71)$. When calculating the odds ratio (OR) that was a 23-fold greater risk of dying when hypoalbuminemia presented compared to the normal albumin group (OR $=23.3 ; 95 \% \mathrm{Cl}: 1,948$ to 279.42$)$. The mean albumin for patients who died was $2.04 \mathrm{~g} / \mathrm{dL}( \pm 0.31)$ vs. $3.03 \mathrm{~g} / \mathrm{dL}( \pm 0.35)(p=0.02 ; 95 \% \mathrm{Cl}:-1.551$ to -0.416$)$. We do not assess morbidity, however, we identify a certain tendency to a longer stay in the ICU which is accompanied by a higher risk of complications and in the end a higher risk of mortality. Conclusion: We conclude that hypoalbuminemia represents a predictor of mortality in patients with abdominal sepsis.
\end{abstract}

Key words: Hypoalbuminemia. Sepsis. Abdominal sepsis.

\section{Correspondencia:}

*Eric M. Saucedo-Moreno

Gobernador Gregorio Villa Gelati, 29

$\begin{array}{ll}\text { Col. San Miguel Chapultepec } 1 \text { Sección, Del. Miguel Hidalgo } & \text { Fecha de recepción: 24-11-2019 } \\ \text { C.P. 11850, Ciudad de México, México } & \text { Fecha de aceptación: 15-01-2020 }\end{array}$

E-mail: cirugía.saucedo@ gmail.com

DOI: $10.24875 / C I R U .20001712$

Cir Cir. 2020;88(4):481-484

Contents available at PubMed

www.cirugiaycirujanos.com

0009-7411/@ 2020 Academia Mexicana de Cirugía. Publicado por Permanyer. Este es un artículo open access bajo la licencia CC BY-NC-ND (http://creativecommons.org/licenses/by-nc-nd/4.0/). 


\section{Introducción}

La concentración sérica normal de albúmina en personas sanas es de aproximadamente $35-50 \mathrm{~g} / \mathrm{l}$. Una cifra $<35 \mathrm{~g} / \mathrm{l}$ de albúmina (hipoalbuminemia) es común en pacientes gravemente enfermos y ha demostrado ser un parámetro para predecir mortalidad y morbilidad ${ }^{1,2}$.

La intención del presente estudio fue conocer la aplicación de la albúmina como predictor de mortalidad en pacientes con sepsis de origen abdominal.

\section{Método}

Realizamos un estudio en un hospital privado en la Ciudad de México de manera prospectiva en un periodo de 12 meses comprendido entre junio de 2018 y junio 2019. Incluimos todos los pacientes de 18 a 95 años con diagnóstico de sepsis de origen abdominal que ingresaron a la unidad de terapia intensiva. Hicimos el diagnóstico de sepsis de origen abdominal de acuerdo con las guías Society of Critical Care Medicine (SCCM) y (ESICM) The European Society of Intensive Care Medicina de 2016: pacientes con sospecha de infección abdominal que presentaron disfunción orgánica caracterizada por 20 más puntos en la escala Sequential Organ Failure Assessment (SOFA) $)^{3}$.

Excluimos a los pacientes trasladados de otra institución, aquellos que recibieron transfusión de albúmina intravenosa antes de la medición de las concentraciones séricas y los que previamente tenían enfermedad renal crónica o hepatopatía. A todos los pacientes les realizamos medición de albúmina extraída por punción o por catéter venoso central, en un tubo con citrato. Dividimos la muestra en dos grupos: grupo 1 con albúmina $>3.5 \mathrm{~g} / \mathrm{dl}$ y grupo 2 con albúmina $<3.4 \mathrm{~g} / \mathrm{dl}$. El seguimiento lo realizamos hasta el egreso del paciente de la terapia intensiva y se comparó la mortalidad entre ambos grupos.

Para variables cuantitativas con distribuciones paramétricas utilizamos la prueba $t$ de Student y los datos se expresaron como media \pm desviación estándar. Utilizamos la prueba de ji al cuadrado o exacta de Fisher para datos categóricos. Consideramos un valor $<0.05$ para una $p$ significativa y su intervalo de confianza del 95\% (IC 95\%). Los datos se analizaron utilizando el software SPSS (versión 20.0 para Windows $)^{4}$.
Tabla 1. Variables generales de la muestra estudiada

\begin{tabular}{lc}
\hline & Pacientes $(\mathbf{n}=\mathbf{2 3})$ \\
\hline Edad, años & $60.87( \pm 18.51)$ \\
Sexo, F/M & $12 / 11(52.2 \%)$ \\
Albúmina, g/dl & $2.77( \pm 0.71)$ \\
Índice de masa corporal & $26.88( \pm 5.28)$ \\
SOFA & $6.13( \pm 4.003)$ \\
APACHE & $19.52( \pm 8.6)$ \\
Procalcitonina & $19.37( \pm 28.13)$ \\
Tiempo de estancia en terapia intensiva, días & $6.83(4.85)$ \\
(desviación estándar) & \\
\hline
\end{tabular}

\section{Resultados}

Estudiamos en total 23 pacientes ingresados a la unidad de terapia intensiva de los cuales el $52.2 \%$ (12) eran de sexo femenino, con una media de edad de 60.87 años $( \pm 3.4)$. La media de los valores de albúmina fue de $2.77 \mathrm{~g} / \mathrm{dl}( \pm 0.71)$ (Tabla 1).

Dividimos la muestra en dos grupos: el grupo 1 $(n=8)$ de pacientes con hipoalbuminemia y el grupo 2 ( $n=15)$ de pacientes con albúmina normal. Al comparar las variables estudiadas observamos que existe homogeneidad entre ambos grupos sin diferencias significativas, excepto para la proporción de pacientes que fallecieron. Al calcular la odds ratio (OR) identificamos un riesgo 23 veces mayor de fallecer al presentar hipoalbuminemia en comparación con el grupo con albúmina normal (OR = 23.3; IC 95\%: 1.948 a 279.42]) (Tabla 2). La media de los valores de albúmina para los pacientes que fallecieron fue de $2.04 \mathrm{~g} / \mathrm{dl}$ $( \pm 0.31)$ vs. $3.03 \mathrm{~g} / \mathrm{dl}( \pm 0.35)$ en los que no fallecieron $(p=0.02$; IC 95\%: -1.551 a -0.416$)$ (Tabla 3 y Fig. 1).

\section{Discusión}

Es bien conocido que los pacientes con diagnóstico de sepsis abdominal, sobre todo aquellos con estancia en la unidad de terapia intensiva, pueden presentar desnutrición o agravarse al menos por tres mecanismos distintos: 1) redistribución al espacio intersticial, 2) pérdida de albúmina durante el procedimiento quirúrgico y 3) aumento del catabolismo. Numerosos estudios han demostrado que unas cifras bajas de albúmina se asocian a mayor morbilidad ${ }^{5}$. 
Tabla 2. Comparación entre grupos

\begin{tabular}{|c|c|c|c|c|}
\hline & $\begin{array}{l}\text { Grupo 1, albúmina normal } \\
\qquad(\mathrm{n}=8)\end{array}$ & $\begin{array}{l}\text { Grupo 2, hipoalbuminemia } \\
\qquad(n=15)\end{array}$ & p & IC 95\% \\
\hline Edad, años & $64.88(21.781)$ & $58.73(16.94)$ & 0.461 & -10.88 a 23.165 \\
\hline Sexo, F/M & $7 / 1$ & $5 / 10$ & 0.27 & \\
\hline Índice de masa corporal & $25.61(3.94)$ & $27.55(5.89)$ & 0.415 & -1.48 a -0.83 \\
\hline SOFA & $7.75(4.97)$ & $5.27(3.2)$ & 0.161 & -1.073 a 6.04 \\
\hline APACHE & $25.25(10.99)$ & $16.46(5.3)$ & 0.17 & 1.77 a 15.74 \\
\hline Procalcitonina & $15.2(22.70)$ & $21.71(31.38)$ & 0.61 & -32.9 a 20.05 \\
\hline $\begin{array}{l}\text { Tiempo de estancia en terapia intensiva } \\
\text { (desviación estándar) }\end{array}$ & $7.25(4.83)$ & $6.60(1.29)$ & 0.768 & -3.86 a 5.168 \\
\hline Vivos/fallecidos & $3(37 \%) / 5(63.5 \%)$ & $1(26.1 \%) / 4(93.3 \%)$ & 0.009 & $\mathrm{OR}=23.31 .948$ a 279.42 \\
\hline
\end{tabular}

IC: intervalo de confianza; OR: odds ratio.

Tabla 3. Comparación de las medias de los valores de albúmina en pacientes fallecidos y vivos

\begin{tabular}{cccc}
\hline $\begin{array}{c}\text { Fallecidos } \\
(\mathrm{n}=6)\end{array}$ & $\begin{array}{c}\text { Vivos } \\
(\mathrm{n}=17)\end{array}$ & $\mathrm{p}$ & IC 95\% \\
\hline
\end{tabular}

Valor de $2.04(0.312) \quad 3.03(0.635) 0.002-1.551$ a -0.416 albúmina, g/dl

IC: intervalo de confianza.

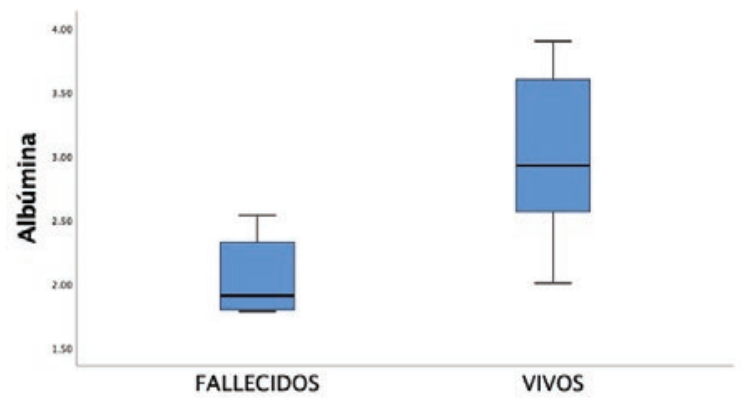

Figura 1. Relación entre los valores de albúmina y la mortalidad. Se aprecia que, a menor nivel de albúmina, mayor mortalidad $(p=0.002)$.

Tambien se ha identificado en diversos estudios que elevados puntajes en escalas como Acute Physiology and Chronic Health disease Classification System (APACHE) y SOFA representan un parámetro confiable para predecir la mortalidad, así como unas concentraciones bajas de albúmina en las primeras 48 horas de ingreso en la unidad de terapia intensiva ${ }^{6}$. Un metaanálisis que incluyó 291,433 pacientes en estado crítico halló que la hipoalbuminemia fue un potente predictor independiente de mortalidad: por $1 \mathrm{~g} / \mathrm{dl}$ que disminuye la albúmina, los riesgos de morbilidad y de mortalidad aumentan un $89 \%$ y un $137 \%$, respectivamente, y la mortalidad se asocia a cerca de un $100 \%$ en pacientes con valores de albúmina $<2 \mathrm{~g} / \mathrm{dl}^{2}$.Nosotros no valoramos la morbilidad, pero identificamos cierta tendencia a un mayor tiempo de estancia en la unidad de terapia intensiva, siendo aventurado concluir que los pacientes presentaron mayor morbilidad considerando que es directamente proporcional a la estancia hospitalaria. De igual forma, identificamos un riesgo 23 veces mayor de mortalidad con valores de albúmina $<2.4 \mathrm{~g} / \mathrm{dl}$ al momento de ingreso al servicio de terapia intensiva.

En los pacientes con sepsis de origen abdominal es característico el aumento de la permeabilidad a las proteínas y a otras macromoléculas en los capilares. Algunos estudios que midieron directamente la permeabilidad a la albúmina demostraron que la velocidad de escape aumenta hasta un 300\%7. La activación de neutrófilos y la liberación de citocinas aumentan esta permeabilidad capilar, disminuyendo las cargas negativas del endotelio y la integridad de las uniones endoteliales. Las toxinas bacterianas, por su parte, reducen el poder oncótico y favorecen las fuerzas osmóticas hacia el intersticio. Se encuentra descrito en la literatura que los pacientes que recuperan sus valores normales de albúmina mejoran su pronóstico ${ }^{8}$. Un estudio realizado por Woods y Kelley ${ }^{9}$ demostró que la distribución de la albúmina posterior a la inyección exógena producía una elevación similar en su concentración plasmática, pero el descenso a los 30 minutos era mayor en los pacientes con sepsis. Estos hallazgos sustentaríanque la reposición de albúmina exógena no ha demostrado beneficio clínicamente significativo $0^{9,10}$. 
En 2013, el estudio multicéntrico EARSS no mostró diferencias significativas en la mortalidad al utilizar de manera exógena albúmina al $20 \%$ en pacientes con sepsis ${ }^{11}$. Desafortunadamente, en nuestro estudio no fue posible comparar el beneficio de utilizar albúmina, secundario a que el costo dificulta su utilidad.

Una de las limitaciones de este estudio, por ser observacional, es que el potencial de error sea alto; sin embargo, se alcanzó suficiente poder estadístico con resultados clínicamente significativos. Otra limitación es que se llevó a cabo en un único centro quirúrgico privado y el tamaño de la muestra es muy pequeño. La metodología es bastante sencilla, pero el resultado tiene un alto impacto clínico y además es aplicable a todos los tipos de cirugía. Son necesarios estudios adicionales con diferentes modelos para determinar la asociación de la hipoalbuminemia con la morbimortalidad en pacientes posoperados.

\section{Conclusiones}

En nuestro estudio obtuvimos un riesgo 23 veces mayor de mortalidad en pacientes con hipoalbuminemia en comparación con aquellos con valores $>2.5 \mathrm{~g} / \mathrm{dl}$. Por ello, concluimos que la albúmina representa un predictor de mortalidad bastante eficiente y que es de muy fácil acceso para todos los hospitales.

\section{Agradecimientos}

Al Servicio de Enseñanza del Hospital Ángeles MOCEL y los residentes de cirugía general.

\section{Conflicto de intereses}

Los autores declaran que no existe conflicto de intereses.

\section{Responsabilidades éticas}

Protección de personas y animales. Los autores declaran que para esta investigación no se han realizado experimentos en seres humanos ni en animales.

Confidencialidad de los datos. Los autores declaran que han seguido los protocolos de su centro de trabajo sobre la publicación de datos de pacientes.

Derecho a la privacidad y consentimiento informado. Los autores han obtenido el consentimiento informado de los pacientes y/o sujetos referidos en el artículo. Este documento obra en poder del autor de correspondencia.

\section{Bibliografía}

1. Jain RK, Chakravorty N, Chakravorty D, Bhattacharya PK, Yadava A, Agarwal RC. Albumin: An overview of its place in current clinical practice. Indian J Anaesth. 2004:48:433-8.

2. Vincent $J$, Dubois $M$, Navickis R, Wilkes MM. Hipoalbuminemia in acute illness: is there a rationale for intervention? A meta-analysis of cohort studies and controlled trials. Ann Surg. 2003;237:319-34.

3. Singer M, Deutschman CS, Seymour CW, Shankar-Hari M, Annane D, Bauer M, et al. The Third International Consensus Definitions for Sepsis and Septic Shock (Sepsis-3). JAMA. 2016;315:801-10.

4. Saucedo EM, Fenig J. Estadística en cirugía. Cómo entender y aplicar conceptos básicos. Cir Cir. 2019;87:692-7.

5. Horsey P. The Cochrane 1998 albumin review - not all it was craked up to be. Euro J Anesth. 2002;19:170-4

6. Blunt M, Nicholson J, Park C. Serum albumin and colloid osmotic pressure in survivors and nonsurvivor of prolonged critical illness. Anesth. 1998:53:755-61.

7. Dubois M, Orellana-Jiménez C, Melot C, De Backer D, Berre J, Leeman $M$, et al. Albumin administration improves organ function in critically ill hypoalbuminemic patients: a prospective, randomized controlled, pilot study. Crit Care Med. 2006;34:2536-40.

8. Finfer S, Norton R, Bellomo R, Boyce N, French J, Myburgh J. The SAFE study: saline vs albumin for fluid resuscitation in the critically ill. Vox Sanguinis. 2004;350:2247-56.

9. Woods M, Kelley H. Oncotic pressure, albumin and ileus: the effect of albumin replacement on postoperative ileus. Am Surg. 1993;59: 758-63.

10. Charpentier J, Mira JP. EARSS Study Group: Efficacy and tolerance of hyperoncotic albumin administration in septic shock patients: The EARSS study. Intensive Care Med. 2011;37(Suppl 1):S115-S0438.

11. Margason M, Soni N. Changes in the serum albumin concentration and volume expanding effects following of bolus of albumin $20 \%$ in septic patients. Br J Anesth. 2004;92:821-6. 